\title{
Experimental determination of compressor map of the DGEN 380 engine compressor using the WESTT CS/BV turbine engine simulator
}

Currently aviation focuses mainlly on increasing the economy and ecology of engines. Production of $\mathrm{NO}_{x}, \mathrm{CO}_{2}$ and $\mathrm{SO}_{2}$ adversaly impacts the environment. Parallel goal to minimize SFC to achieve both lower: emission and mission costs. The optimization of components is thus very important. One of the ways of optimizing cycle is doing that based on compressor maps. However it is very expensive to plot one since experimental work needs to be done. The aim of this article is to present a methodology of creating compressor map based on ENGINE ANALOGY. There was used the virtual bench WESTT CS/BV for tests to receive pressure ratio and mass flow of DGEN 380 for three different values of flight speed and altitude, while the rotational speed was changed. The construction similarity of CFM 56-5B and APS 3200 gives the opportunity to plotted compressor maps using the engine analogy without the need for an experiment or using the virtual bench.

Key words: compressor map, engine bench, turbofan jet engine, turbine engine, mass flow rate

\section{Introduction}

The aim of this article is to present a methodology of creating compressor map based on engine analogy. This alternative is using given data of any engine to plotted compressor map based on ready to use characteristic of another engine. Before this method will be described first it is worth to mention there is a huge number of methods for obtain compressor map.

In a multi-stage process of turbine engine design the mathematical modeling of its parameters is used. It starts with simply thermo-gas dynamic calculations and ends with $3 \mathrm{D}$ analysis of flow through the components. During the design phase of compressor its characteristic is important. Creating compressor map based on test data is costly and time consuming. It is also important to be aware that these characteristics are really error prone. Specific overview of curve fitting methods for a centrifugal compressor and turbine characteristics is presented by Moraal and Kolmanovsky [9].

There are many methods of determining characteristic and one of them is cubic approximation for speed lines from the Moore Greitzer Model used in article of Drummond and Davison [2].

The second possibility is scaling procedure described by Kurzke and Riegler [4]. This method uses the similarity of the pressure ratio value of the existing map and the new one that will be plotted.

Another way to changing the line of characteristic is modification of diffuser geometric or variable guide vanes which is described by Hunzinker, Dickmann and Emmrich [3]. This also gives the inexpensive to manufacture and compact design.

Availability and accuracy of compressor map allowed calculating the loads of turbine. Kurzke [5] mentions that one percent error in calculations in fan or HP compressor efficiency provides to one percent error in specific fuel consumption of a turbofan. The engine manufacturers reveal only a part of information that is why data collection of components, which are necessary to build model of engine to make this kind of simulation, is impossible.
Due to fact that modeling of compressor map is problematic there is a lot of articles and diploma thesis that dealing with this problem. Ismail and Bhinder [6] used tables to preserve the shape of characteristics as linear and Lagrangian interpolation technique to determine the values of the performance parameters for an arbitrarily selected point on the compressor map. El-Gammal in 1991 publicized diploma in which he described, developed by himself, the criteria and algorithm for a compressor characteristic for linear model [7]. For non-linear models Sieros, Stamatis and Mathioudakis [8] used analytical functions to model map of different types of design. They were even used a genetic algorithm to determine the unknown coefficients of third-order equations relating the mass flow, the pressure ratio, and the isentropic efficiency by Kong, Kho and $\mathrm{Ki}$ [10, 11]. Gravdahl and Egeland [12] described an analytical method to approximate the compressor characteristic based on energy considerations and geometry of the compressor. Their method modifies the ideal characteristic and including friction losses. Mentioned ideas were presented by Gravdahl, Willems, Jager and Egeland as experimental validation [13].

In this article is described another yet methods of plotted compressor map. Engine analogy is an alternative, which allows in a simply way, to get necessary information about engine components and their operating range.

The turbine engines are used as propulsion of airplane. The main construction with one duct consists of: inlet, compressor, combustion chamber, turbine and nozzle through which air flows. During the development of more efficiency ones, there is a turbofan with high-bypass ratio. This construction has a fan with huge diameter between the inlet and the compressor and divided duct: internal and external one, which allows to get more thrust.

\section{Compressor map}

\subsection{Use of compressor map}

Another way to get engine with high efficiency is using the compressor map. It allows finding the best conditions of compressor cooperation with the other components and shows stall margin. During the engine crosses mentioned 
stall margin, there is dangerous situation, in which the hot flow begin moving in the opposite direction getting through the blade clearance and this results in air is sucked from the combustion chamber.

The parameters using to plotting the characteristic are reduced parameter, which are closely related with compressor performance. Reducing of parameters is necessary, because then the independence of the characteristic from environmental conditions is obtained. The change of ambient temperature causes the change of mass flow, pressure ratio and efficiency.

The compressor maps may be compared with each other only in the case of similar construction. That means map of axial compressor could be compared only with another axial compressor, not with centrifugal one. The characteristic of radial compressor is milder then characteristic of axial compressor. It means that axial compressor is more sensitive to mass flow changing. Especially dangerous is decrease of mass flow compared with the calculated value, because then occurs boundary-layer separation what results the vortex field. In this situation the pressure is starting to be lower in the component and causes the appearance of swirl between blade space in external duct, pressure pulsation and decrease of efficiency which all make up the compressor unstable work.

The characteristic of axial and radial compressor are on Fig. 1 and Fig. 2.

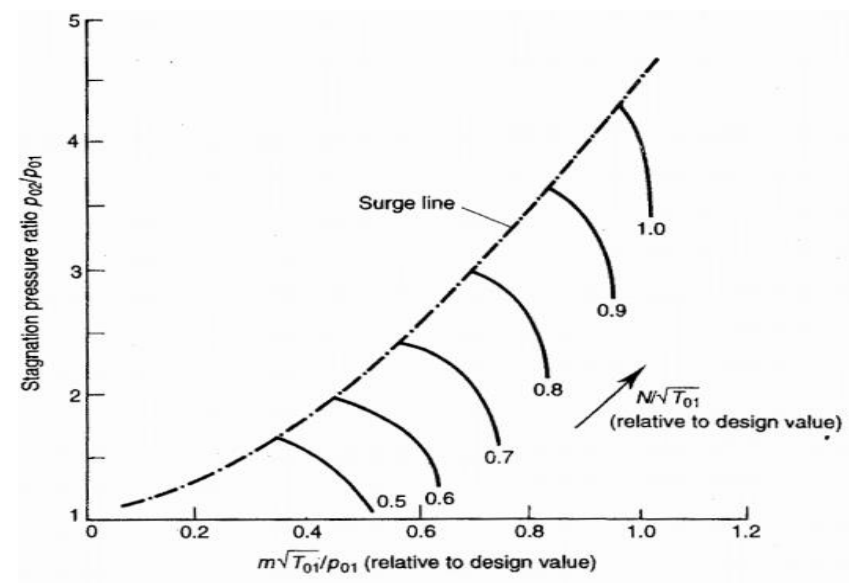

Fig. 1. Axial compressor map with reduced parameters [1]

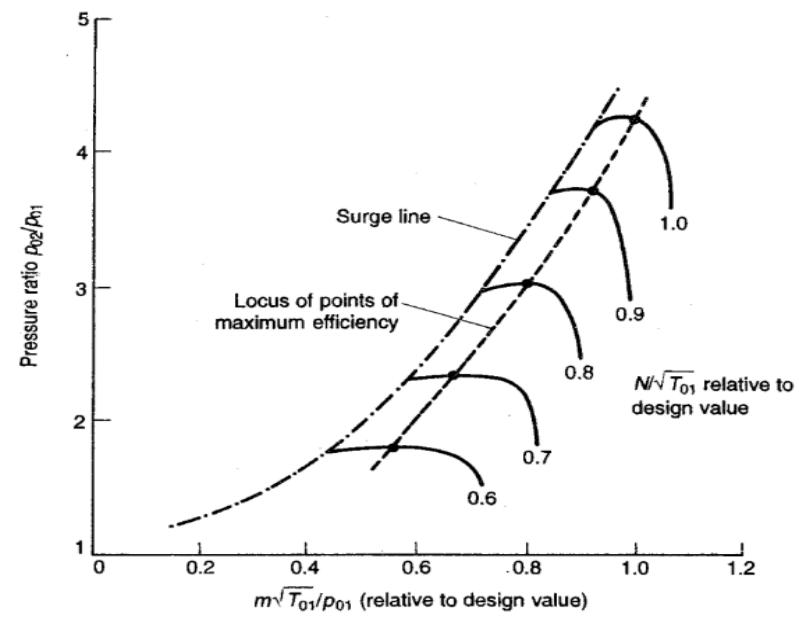

Fig. 2. Centrifugal compressor map with reduced parameters [1]

\subsection{Receiving a compressor map}

Through the use of virtual engine test bench WESTT $\mathrm{CS} / \mathrm{BV}$ which is a simulator of DGEN 380 engine, there was the opportunity to get data like: efficiency and pressure ratio for fan and compressor. All parameters were getting for flight velocity $\mathrm{V}=0 \mathrm{Ma}, \mathrm{V}=0.2 \mathrm{Ma}$ and $\mathrm{V}=0.44 \mathrm{Ma}$ and also for flight altitude $\mathrm{H}=0 \mathrm{~m}, \mathrm{H}=2000 \mathrm{~m}$ and $\mathrm{H}=7000 \mathrm{~m}$.

Virtual test bench allows simulating the work of engine through the change of internal parameters like: pressure and temperature in inlet but the collected parameters had to be reduced.

Another way to get compressor map is apply engine analogy. Plotted characteristic of DGEN 380 may be using for plotting compressor map of another engine provided that the construction of component is similar.

\subsection{DGEN 380, APS 3200, CFM 56-5B- data}

The choice of DGEN 380 was imposed by virtual engine test bench, which is available in Rzeszow University of Technology in Department of Aircrafts and Aircraft Engines. However the APS 3200 and the CFM 56-5B was not random choice. Mentioned engine analogy to be used correctly requires the preservation of construction similarity. The construction of fan in CFM 56-5B is similar to construction of fan in DGEN 380 and the construction of compressor in APS 3200 is the same in DGEN 380- both are centrifugal one.

Table 1.Parameter of DGEN 380

\begin{tabular}{|c|c|c|c|}
\hline Engine & Parameter & Unit & Value \\
\hline \multirow{2}{*}{ DGEN 380 } & Mass flow & $\frac{\mathrm{kg}}{\mathrm{s}}$ & 13 \\
\cline { 2 - 4 } & Pressure ratio & - & 5.3 \\
\hline \multirow{3}{*}{ APS 3200 } & Mass flow & $\frac{\mathrm{kg}}{\mathrm{s}}$ & 2.2 \\
\cline { 2 - 4 } & Pressure ratio & - & 8 \\
\hline \multirow{2}{*}{ CFM 56-5B } & Mass flow & $\frac{\mathrm{kg}}{\mathrm{s}}$ & 400 \\
\cline { 2 - 4 } & Pressure ratio & - & 1.6 \\
\hline
\end{tabular}

\subsection{Preparation of data}

As mentioned the compressor map is plotted in reduced parameters. That is way the charts have got reduced mass flow. To get that the equation (1) was used.

$$
\dot{\mathrm{m}}_{\mathrm{r}}=\dot{\mathrm{m}} \cdot \frac{101325}{\mathrm{p}_{0}} \cdot \sqrt{\frac{\mathrm{T}_{0}}{288.15}}
$$

Based on reduced mass flow and pressure ratio the points have been plotted on the chart. Both charts are for components of DGEN 380 for three different altitudes $(0 \mathrm{~m}$, $2000 \mathrm{~m}, 7000 \mathrm{~m}$ ) and velocities (0 Ma, 0.2 Ma, 0.44 Ma) of flight.

Can be observed lines of centrifugal compressor (Fig. 4) of DGEN 380 do not show characteristic division into three groups like the lines of fan of DGEN 380 (Fig. 3). This is due to the fact that flow in the compressor is more stable because of the fan preceding it and stabilizes parameters of flow. The similarity of these lines is caused by the centrifugal compressor show less susceptibility to crossed stall margin. 


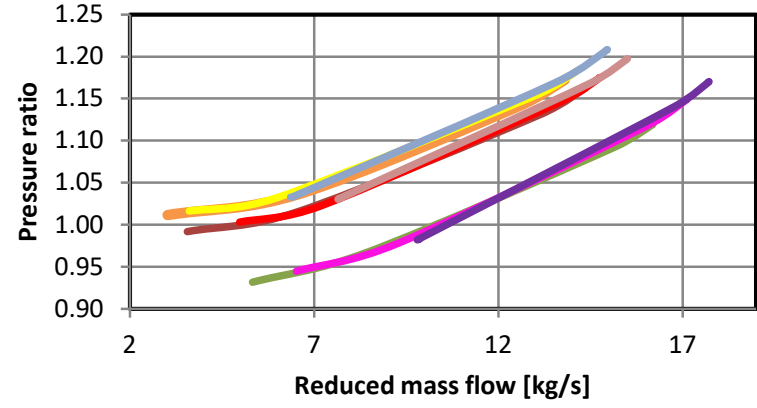

$\begin{array}{lll}\longrightarrow \mathrm{V}=0 \mathrm{Ma} ; \mathrm{H}=\mathrm{Om} & -\mathrm{V}=0 \mathrm{Ma} ; \mathrm{H}=2000 \mathrm{~m} & -\mathrm{V}=0 \mathrm{Ma} ; \mathrm{H}=7000 \mathrm{~m} \\ \mathrm{~V}=0,2 \mathrm{Ma} ; \mathrm{H}=0 \mathrm{~m} & -\mathrm{V}=0,2 \mathrm{Ma} ; \mathrm{H}=2000 \mathrm{~m} & -\mathrm{V}=0,2 \mathrm{Ma} ; \mathrm{H}=7000 \mathrm{~m} \\ \mathrm{~V}=0,44 \mathrm{Ma} ; \mathrm{H}=0 \mathrm{~m} & \longrightarrow \mathrm{V}=0,44 \mathrm{Ma} ; \mathrm{H}=2000 \mathrm{~m} & -\mathrm{V}=0,44 \mathrm{Ma} ; \mathrm{H}=7000 \mathrm{~m}\end{array}$

Fig. 3. Compressor map for fan of DGEN 380

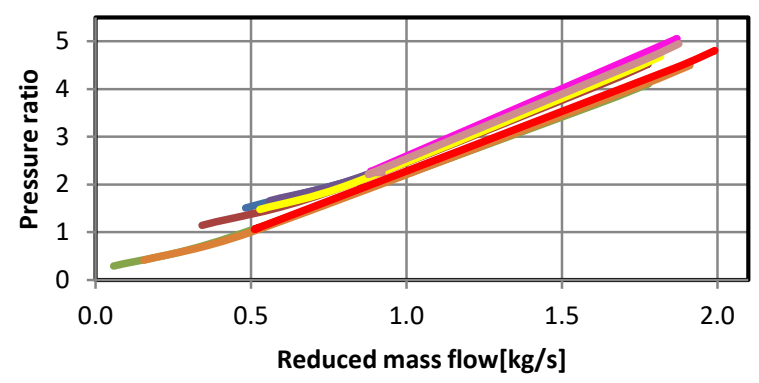

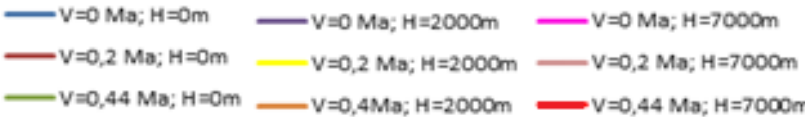

Fig. 4. Compressor map for centrifugal compressor of DGEN 380

\subsection{Engine analogy}

As was mentioned before, there is possibility to plot compressor map of another engine based on obtained compressor map DGEN 380 only if similarity of construction is preserved. It is called engine analogy.

The first step was determined absolute parameters of pressure ratio and mass flow. Absolute parameter of pressure ratio is value of pressure ratio read when collecting data is divided by maximal value of sea-level pressure ratio. While absolute parameter of mass flow is value of mass flow read when collecting data is divided by maximal value of sea-level mass flow. The equation (2) and (3) was used to carry out such calculation.

$$
\begin{aligned}
\pi_{\mathrm{abs}} & =\frac{\pi}{\pi_{\max }} \\
\dot{\mathrm{m}}_{\mathrm{abs}} & =\frac{\dot{\mathrm{m}}}{\dot{\mathrm{m}}_{\max }}
\end{aligned}
$$

Each absolute parameter need to be multiply by pressure ratio or mass flow of suitable engine and then may be plotted. To get all of the points to plotted compressor map of fan CFM 56-5B and compressor APS 3200 there was wrote the program on MATLAB.

For CFM 56-5B obtained parameters by using program, are showed in Tables 4-12. All of them are determined for different rotational velocity and it can be seen despite the change of the value of rotational velocity the parameters on the last rows are identical or the same.
Table 4. Parameter of CFM 56-5B V = $0 \mathrm{Ma}, \mathrm{H}=0 \mathrm{~m}$

\begin{tabular}{|l|c|c|c|c|c|c|}
\hline & $\begin{array}{c}\text { Pres- } \\
\text { sure } \\
\text { ratio }\end{array}$ & $\begin{array}{c}\text { Reduced } \\
\text { mass flow }\end{array}$ & $\begin{array}{c}\text { Absolute } \\
\text { pressure } \\
\text { ratio }\end{array}$ & $\begin{array}{c}\text { CFM 56-5B } \\
\text { fan pressure } \\
\text { ratio }\end{array}$ & $\begin{array}{c}\text { Absolute } \\
\text { mass } \\
\text { flow }\end{array}$ & $\begin{array}{c}\text { CFM 56- } \\
\text { 5B fan } \\
\text { mass flow }\end{array}$ \\
\hline Idle-0\% & 1.01 & 3.03 & 0.86 & 1.38 & 0.22 & 87.83 \\
\hline $30 \%$ & 1.04 & 6.64 & 0.89 & 1.42 & 0.48 & 192.46 \\
\hline Cruise- 43\% & 1.14 & 12.52 & 0.97 & 1.56 & 0.91 & 362.90 \\
\hline $\begin{array}{l}\text { Max Cont.- } \\
74 \%\end{array}$ & 1.17 & 13.77 & 1.00 & 1.60 & 1.00 & 399.13 \\
\hline Top-100\% & 1.17 & 13.8 & 1.00 & 1.60 & 1.00 & 400.00 \\
\hline
\end{tabular}

Table 5. Parameter of CFM 56-5B V = 0.2 Ma, $\mathrm{H}=0 \mathrm{~m}$

\begin{tabular}{|l|c|c|c|c|c|c|}
\hline & $\begin{array}{c}\text { Pres- } \\
\text { sure } \\
\text { ratio }\end{array}$ & $\begin{array}{c}\text { Reduced } \\
\text { mass flow }\end{array}$ & $\begin{array}{c}\text { Absolute } \\
\text { pressure } \\
\text { ratio }\end{array}$ & $\begin{array}{c}\text { CFM 56-5B } \\
\text { fan pressure } \\
\text { ratio }\end{array}$ & $\begin{array}{c}\text { Absolute } \\
\text { mass } \\
\text { flow }\end{array}$ & $\begin{array}{c}\text { CFM 56- } \\
\text { 5B fan } \\
\text { mass flow }\end{array}$ \\
\hline Idle-0\% & 0.99 & 3.56 & 0.85 & 1.35 & 0.26 & 103.19 \\
\hline $30 \%$ & 1.02 & 6.59 & 0.87 & 1.39 & 0.48 & 191.01 \\
\hline Cruise- 43\% & 1.13 & 13.14 & 0.97 & 1.55 & 0.95 & 380.87 \\
\hline $\begin{array}{l}\text { Max Cont.- } \\
74 \%\end{array}$ & 1.16 & 14.37 & 0.99 & 1.59 & 1.04 & 416.52 \\
\hline Top-100\% & 1.16 & 14.37 & 0.99 & 1.59 & 1.04 & 416.52 \\
\hline
\end{tabular}

Table 6. Parameter of CFM 56-5B V = 0.44 Ma, $\mathrm{H}=0 \mathrm{~m}$

\begin{tabular}{|l|c|c|c|c|c|c|}
\hline & $\begin{array}{c}\text { Pres- } \\
\text { sure } \\
\text { ratio }\end{array}$ & $\begin{array}{c}\text { Reduced } \\
\text { mass flow }\end{array}$ & $\begin{array}{c}\text { Absolute } \\
\text { pressure } \\
\text { ratio }\end{array}$ & $\begin{array}{c}\text { CFM 56-5B } \\
\text { fan pressure } \\
\text { ratio }\end{array}$ & $\begin{array}{c}\text { Absolute } \\
\text { mass } \\
\text { flow }\end{array}$ & $\begin{array}{c}\text { CFM 56- } \\
\text { 5B fan } \\
\text { mass flow }\end{array}$ \\
\hline Idle-0\% & 0.93 & 5.33 & 0.79 & 1.27 & 0.39 & 154.49 \\
\hline $30 \%$ & 0.97 & 8.49 & 0.83 & 1.33 & 0.62 & 246.09 \\
\hline Cruise- 43\% & 1.09 & 15.15 & 0.9 & 1.49 & 1.10 & 439.13 \\
\hline $\begin{array}{l}\text { Max Cont.- } \\
74 \%\end{array}$ & 1.12 & 16.2 & 0.96 & 1.53 & 1.17 & 469.57 \\
\hline Top-100\% & 1.12 & 16.19 & 0.96 & 1.53 & 1.17 & 469.28 \\
\hline
\end{tabular}

Table 7. Parameter of CFM 56-5B V =0 Ma, H=2000 m

\begin{tabular}{|l|c|c|c|c|c|c|}
\hline & $\begin{array}{c}\text { Pres- } \\
\text { sure } \\
\text { ratio }\end{array}$ & $\begin{array}{c}\text { Reduced } \\
\text { mass flow }\end{array}$ & $\begin{array}{c}\text { Absolute } \\
\text { pressure } \\
\text { ratio }\end{array}$ & $\begin{array}{c}\text { CFM 56-5B } \\
\text { fan pressure } \\
\text { ratio }\end{array}$ & $\begin{array}{c}\text { Absolute } \\
\text { mass } \\
\text { flow }\end{array}$ & $\begin{array}{c}\text { CFM 56- } \\
\text { 5B fan } \\
\text { mass flow }\end{array}$ \\
\hline Idle-0\% & 1.02 & 3.61 & 0.87 & 1.39 & 0.26 & 104.64 \\
\hline $30 \%$ & 1.03 & 6.00 & 0.88 & 1.41 & 0.43 & 173.91 \\
\hline Cruise- 43\% & 1.15 & 13.06 & 0.98 & 1.57 & 0.95 & 378.55 \\
\hline $\begin{array}{l}\text { Max Cont.- } \\
74 \%\end{array}$ & 1.18 & 14.12 & 1.01 & 1.61 & 1.02 & 409.28 \\
\hline Top-100\% & 1.18 & 14.12 & 1.01 & 1.61 & 1.02 & 409.28 \\
\hline
\end{tabular}

Table 8. Parameter of CFM 56-5B V =0.2 Ma, $\mathrm{H}=2000 \mathrm{~m}$

\begin{tabular}{|l|c|c|c|c|c|c|}
\hline & $\begin{array}{c}\text { Pressure } \\
\text { ratio }\end{array}$ & $\begin{array}{c}\text { Reduced } \\
\text { mass flow }\end{array}$ & $\begin{array}{c}\text { Absolute } \\
\text { pressure } \\
\text { ratio }\end{array}$ & $\begin{array}{c}\text { CFM 56-5B } \\
\text { fan pressure } \\
\text { ratio }\end{array}$ & $\begin{array}{c}\text { Absolute } \\
\text { mass } \\
\text { flow }\end{array}$ & $\begin{array}{c}\text { CFM 56- } \\
\text { 5B fan } \\
\text { mass flow }\end{array}$ \\
\hline Idle-0\% & 1.00 & 4.98 & 0.85 & 1.37 & 0.36 & 144.35 \\
\hline $30 \%$ & 1.02 & 7.20 & 0.87 & 1.39 & 0.52 & 208.70 \\
\hline Cruise- 43\% & 1.15 & 13.76 & 0.98 & 1.57 & 1.00 & 398.84 \\
\hline $\begin{array}{l}\text { Max Cont.- } \\
74 \%\end{array}$ & 1.18 & 14.76 & 1.01 & 1.61 & 1.07 & 427.83 \\
\hline Top-100\% & 1.17 & 14.75 & 1.00 & 1.60 & 1.07 & 427.54 \\
\hline
\end{tabular}

Table 9. Parameter of CFM 56-5B V =0.44 Ma, $\mathrm{H}=2000 \mathrm{~m}$

\begin{tabular}{|l|c|c|c|c|c|c|}
\hline & $\begin{array}{c}\text { Pressure } \\
\text { ratio }\end{array}$ & $\begin{array}{c}\text { Reduced } \\
\text { mass flow }\end{array}$ & $\begin{array}{c}\text { Absolute } \\
\text { pressure } \\
\text { ratio }\end{array}$ & $\begin{array}{c}\text { CFM 56-5B } \\
\text { fan pressure } \\
\text { ratio }\end{array}$ & $\begin{array}{c}\text { Absolute } \\
\text { mass flow }\end{array}$ & $\begin{array}{c}\text { CFM 56- } \\
\text { 5B fan } \\
\text { mass flow }\end{array}$ \\
\hline Idle-0\% & 0.94 & 6.52 & 0.80 & 1.29 & 0.47 & 188.99 \\
\hline $30 \%$ & 0.98 & 9.29 & 0.84 & 1.34 & 0.67 & 269.28 \\
\hline Cruise- 43\% & 1.12 & 16.03 & 0.96 & 1.53 & 1.16 & 464.64 \\
\hline $\begin{array}{l}\text { Max Cont.- } \\
74 \%\end{array}$ & 1.15 & 17.13 & 0.98 & 1.57 & 1.24 & 496.52 \\
\hline Top-100\% & 1.15 & 17.13 & 0.98 & 1.57 & 1.24 & 496.52 \\
\hline
\end{tabular}

Table 10. Parameter of CFM 56-5B V $=0 \mathrm{Ma}, \mathrm{H}=7000 \mathrm{~m}$

\begin{tabular}{|l|c|c|c|c|c|c|}
\hline & $\begin{array}{c}\text { Pressure } \\
\text { ratio }\end{array}$ & $\begin{array}{c}\text { Reduced } \\
\text { mass flow }\end{array}$ & $\begin{array}{c}\text { Absolute } \\
\text { pressure } \\
\text { ratio }\end{array}$ & $\begin{array}{c}\text { CFM 56-5B } \\
\text { fan pressure } \\
\text { ratio }\end{array}$ & $\begin{array}{c}\text { Absolute } \\
\text { mass flow }\end{array}$ & $\begin{array}{c}\text { CFM 56- } \\
\text { 5B fan } \\
\text { mass flow }\end{array}$ \\
\hline Idle-0\% & 1.03 & 6.37 & 0.88 & 1.41 & 0.46 & 184.64 \\
\hline $30 \%$ & 1.03 & 6.43 & 0.88 & 1.41 & 0.47 & 186.38 \\
\hline Cruise- 43\% & 1.17 & 13.58 & 1.00 & 1.60 & 0.98 & 393.62 \\
\hline $\begin{array}{l}\text { Max Cont.- } \\
74 \%\end{array}$ & 1.21 & 14.96 & 1.03 & 1.65 & 1.08 & 433.62 \\
\hline Top-100\% & 1.21 & 14.96 & 1.03 & 1.65 & 1.08 & 433.62 \\
\hline
\end{tabular}


Experimental determination of compressor map of the DGEN 380 compressor using the WESTT CS/BV turbine engine simulation

Table 11. Parameter of CFM 56-5B V =0.2 Ma, $\mathrm{H}=7000 \mathrm{~m}$

\begin{tabular}{|l|c|c|c|c|c|c|}
\hline & $\begin{array}{c}\text { Pressure } \\
\text { ratio }\end{array}$ & $\begin{array}{c}\text { Reduced } \\
\text { mass flow }\end{array}$ & $\begin{array}{c}\text { Absolute } \\
\text { pressure } \\
\text { ratio }\end{array}$ & $\begin{array}{c}\text { CFM 56-5B } \\
\text { fan pressure } \\
\text { ratio }\end{array}$ & $\begin{array}{c}\text { Absolute } \\
\text { mass } \\
\text { flow }\end{array}$ & $\begin{array}{c}\text { CFM 56- } \\
\text { 5B fan } \\
\text { mass flow }\end{array}$ \\
\hline Idle-0\% & 1.03 & 7.74 & 0.80 & 1.41 & 0.56 & 224.35 \\
\hline $30 \%$ & 1.03 & 7.65 & 0.80 & 1.41 & 0.55 & 221.74 \\
\hline Cruise- 43\% & 1.17 & 14.47 & 0.86 & 1.60 & 1.05 & 419.42 \\
\hline $\begin{array}{l}\text { Max Cont.- } \\
74 \%\end{array}$ & 1.20 & 15.50 & 0.88 & 1.64 & 1.12 & 449.28 \\
\hline Top-100\% & 1.20 & 15.50 & 0.88 & 1.64 & 1.12 & 449.28 \\
\hline
\end{tabular}

Table 12. Parameter of CFM 56-5B V =0.44 Ma, $\mathrm{H}=7000 \mathrm{~m}$

\begin{tabular}{|l|c|c|c|c|c|c|}
\hline & $\begin{array}{c}\text { Pressure } \\
\text { ratio }\end{array}$ & $\begin{array}{c}\text { Reduced } \\
\text { mass flow }\end{array}$ & $\begin{array}{c}\text { Absolute } \\
\text { pressure } \\
\text { ratio }\end{array}$ & $\begin{array}{c}\text { CFM 56-5B } \\
\text { fan pressure } \\
\text { ratio }\end{array}$ & $\begin{array}{c}\text { Absolute } \\
\text { mass flow }\end{array}$ & $\begin{array}{c}\text { CFM 56- } \\
\text { 5B fan } \\
\text { mass flow }\end{array}$ \\
\hline Idle-0\% & 0.98 & 9.86 & 0.60 & 1.34 & 0.71 & 285.80 \\
\hline $30 \%$ & 0.98 & 9.80 & 0.60 & 1.34 & 0.71 & 284.06 \\
\hline Cruise- 43\% & 1.14 & 16.78 & 0.80 & 1.56 & 1.22 & 486.38 \\
\hline $\begin{array}{l}\text { Max Cont.- } \\
74 \%\end{array}$ & 1.17 & 17.72 & 0.84 & 1.60 & 1.28 & 513.62 \\
\hline Top-100\% & 1.17 & 17.72 & 0.84 & 1.60 & 1.28 & 513.62 \\
\hline
\end{tabular}

All obtained parameters are plotted in Fig. 5.

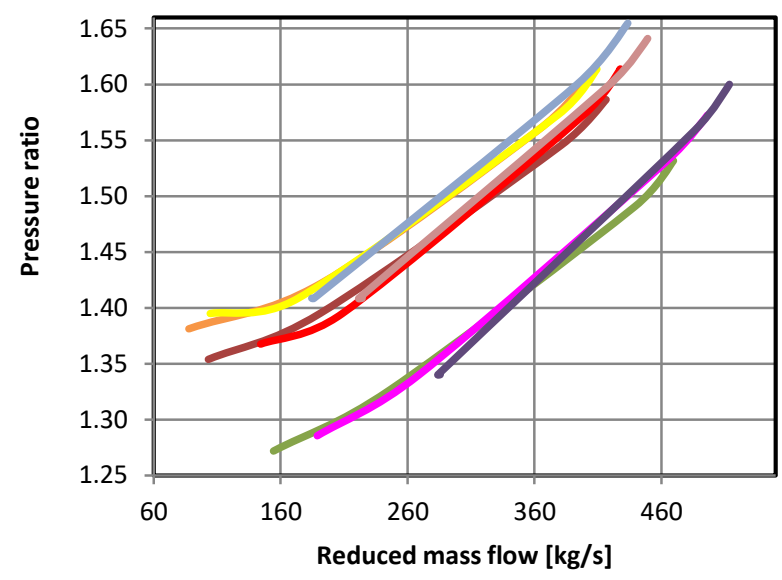

$\begin{array}{cll}\longrightarrow V=0 \mathrm{Ma} ; \mathrm{H}=\mathrm{Om} & -\mathrm{V}=0 \mathrm{Ma} ; \mathrm{H}=2000 \mathrm{~m} & -\mathrm{V}=0 \mathrm{Ma} ; \mathrm{H}=7000 \mathrm{~m} \\ \mathrm{~V}=0,2 \mathrm{Ma} ; \mathrm{H}=\mathrm{Om} & -\mathrm{V}=0,2 \mathrm{Ma} ; \mathrm{H}=2000 \mathrm{~m} & -\mathrm{V}=0,2 \mathrm{Ma} ; \mathrm{H}=7000 \mathrm{~m} \\ \mathrm{~V}=0,44 \mathrm{Ma} ; \mathrm{H}=0 \mathrm{~m} & -\mathrm{V}=0,44 \mathrm{Ma} ; \mathrm{H}=2000 \mathrm{~m} & -\mathrm{V}=0,44 \mathrm{Ma} ; \mathrm{H}=7000 \mathrm{~m}\end{array}$

Fig. 5. Compressor map of fan CFM 56-56

The compressor map of fan CFM 56-5B has got the characteristic division into three groups like the lines of fan of DGEN 380 are present. It is caused by unstable parameters of flow, exactly like in the compressor map of DGEN 380 fan.

For APS 3200 obtained parameters by using program, are showed in Tables 13-21. As in the CFM 56-5B the all of them are determined for different rotational velocity. In this case can be seen the parameters on the last two rows are also identical or the same despite the change of value of rotational.

Table 13. Parameter of APS $3200 \mathrm{~V}=0 \mathrm{Ma}, \mathrm{H}=0 \mathrm{~m}$

\begin{tabular}{|l|c|c|c|c|c|c|}
\hline & $\begin{array}{c}\text { Pressure } \\
\text { ratio }\end{array}$ & $\begin{array}{c}\text { Reduced } \\
\text { mass flow }\end{array}$ & $\begin{array}{c}\text { Absolute } \\
\text { pressure } \\
\text { ratio }\end{array}$ & $\begin{array}{c}\text { APS 3200 } \\
\text { fan pressure } \\
\text { ratio }\end{array}$ & $\begin{array}{c}\text { Absolute } \\
\text { mass flow }\end{array}$ & $\begin{array}{c}\text { APS 3200 } \\
\text { fan mass } \\
\text { flow }\end{array}$ \\
\hline Idle-0\% & 1.51 & 0.48 & 0.33 & 2.63 & 0.27 & 0.60 \\
\hline $30 \%$ & 2.38 & 0.96 & 0.52 & 4.15 & 0.55 & 1.20 \\
\hline Cruise- 43\% & 4.18 & 1.62 & 0.91 & 7.29 & 0.92 & 2.02 \\
\hline $\begin{array}{l}\text { Max Cont.- } \\
74 \%\end{array}$ & 4.58 & 1.76 & 1.00 & 7.98 & 1.00 & 2.19 \\
\hline Top-100\% & 4.59 & 1.76 & 1.00 & 8.00 & 1.00 & 2.20 \\
\hline
\end{tabular}

Table 14. Parameter of APS $3200 \mathrm{~V}=0.2 \mathrm{Ma}, \mathrm{H}=0 \mathrm{~m}$

\begin{tabular}{|l|c|c|c|c|c|c|}
\hline & $\begin{array}{c}\text { Presure } \\
\text { ratio }\end{array}$ & $\begin{array}{c}\text { Reduced } \\
\text { mass flow }\end{array}$ & $\begin{array}{c}\text { Absolute } \\
\text { pressure } \\
\text { ratio }\end{array}$ & $\begin{array}{c}\text { APS 3200 } \\
\text { fan pressure } \\
\text { ratio }\end{array}$ & $\begin{array}{c}\text { Absolute } \\
\text { mass flow }\end{array}$ & $\begin{array}{c}\text { APS 3200 } \\
\text { fan mass } \\
\text { flow }\end{array}$ \\
\hline Idle-0\% & 1.14 & 0.34 & 0.25 & 1.99 & 0.19 & 0.43 \\
\hline $30 \%$ & 1.84 & 0.75 & 0.40 & 3.21 & 0.42 & 0.93 \\
\hline Cruise- 43\% & 4.11 & 1.63 & 0.90 & 7.17 & 0.93 & 2.04 \\
\hline $\begin{array}{l}\text { Max Cont.- } \\
74 \%\end{array}$ & 4.52 & 1.78 & 0.98 & 7.88 & 1.01 & 2.22 \\
\hline Top-100\% & 4.52 & 1.78 & 0.98 & 7.88 & 1.01 & 2.22 \\
\hline
\end{tabular}

Table 15. Parameter of APS $3200 \mathrm{~V}=0.44 \mathrm{Ma}, \mathrm{H}=0 \mathrm{~m}$

\begin{tabular}{|l|c|c|c|c|c|c|}
\hline & $\begin{array}{c}\text { Pressure } \\
\text { ratio }\end{array}$ & $\begin{array}{c}\text { Reduced } \\
\text { mass flow }\end{array}$ & $\begin{array}{c}\text { Absolute } \\
\text { pressure } \\
\text { ratio }\end{array}$ & $\begin{array}{c}\text { APS 3200 } \\
\text { fan pressure } \\
\text { ratio }\end{array}$ & $\begin{array}{c}\text { Absolute } \\
\text { mass flow }\end{array}$ & $\begin{array}{c}\text { APS 3200 } \\
\text { fan mass } \\
\text { flow }\end{array}$ \\
\hline Idle-0\% & 0.29 & 0.06 & 0.06 & 0.51 & 0.03 & 0.07 \\
\hline $30 \%$ & 0.89 & 0.43 & 0.19 & 1.54 & 0.24 & 0.54 \\
\hline Cruise- 43\% & 3.70 & 1.62 & 0.81 & 6.45 & 0.92 & 2.02 \\
\hline $\begin{array}{l}\text { Max Cont.- } \\
74 \%\end{array}$ & 4.11 & 1.78 & 0.90 & 7.16 & 1.01 & 2.22 \\
\hline Top-100\% & 4.11 & 1.78 & 0.89 & 7.16 & 1.01 & 2.22 \\
\hline
\end{tabular}

Table 16. Parameter of APS $3200 \mathrm{~V}=0 \mathrm{Ma}, \mathrm{H}=2000 \mathrm{~m}$

\begin{tabular}{|l|c|c|c|c|c|c|}
\hline & $\begin{array}{c}\text { Pressure } \\
\text { ratio }\end{array}$ & $\begin{array}{c}\text { Reduced } \\
\text { mass flow }\end{array}$ & $\begin{array}{c}\text { Absolute } \\
\text { pressure } \\
\text { ratio }\end{array}$ & $\begin{array}{c}\text { APS 3200 } \\
\text { fan pressure } \\
\text { ratio }\end{array}$ & $\begin{array}{c}\text { Absolute } \\
\text { mass flow }\end{array}$ & $\begin{array}{c}\text { APS 3200 } \\
\text { fan mass } \\
\text { flow }\end{array}$ \\
\hline Idle-0\% & 1.66 & 0.45 & 0.36 & 2.89 & 0.26 & 0.56 \\
\hline $30 \%$ & 2.22 & 0.70 & 0.48 & 3.87 & 0.40 & 0.88 \\
\hline Cruise- 43\% & 4.36 & 1.34 & 0.95 & 7.60 & 0.76 & 1.67 \\
\hline $\begin{array}{l}\text { Max Cont.- } \\
74 \%\end{array}$ & 4.71 & 1.43 & 1.03 & 8.21 & 0.81 & 1.79 \\
\hline Top-100\% & 4.71 & 1.43 & 1.03 & 8.21 & 0.81 & 1.79 \\
\hline
\end{tabular}

Table 17. Parameter of APS $3200 \mathrm{~V}=0.2 \mathrm{Ma}, \mathrm{H}=2000 \mathrm{~m}$

\begin{tabular}{|l|c|c|c|c|c|c|}
\hline & $\begin{array}{c}\text { Pressure } \\
\text { ratio }\end{array}$ & $\begin{array}{c}\text { Reduced } \\
\text { mass } \\
\text { flow }\end{array}$ & $\begin{array}{c}\text { Absolute } \\
\text { pressure } \\
\text { ratio }\end{array}$ & $\begin{array}{c}\text { APS 3200 } \\
\text { fan pressure } \\
\text { ratio }\end{array}$ & $\begin{array}{c}\text { Absolute } \\
\text { mass flow }\end{array}$ & $\begin{array}{c}\text { APS 3200 } \\
\text { fan mass } \\
\text { flow }\end{array}$ \\
\hline Idle-0\% & 1.48 & 0.42 & 0.32 & 2.57 & 0.24 & 0.53 \\
\hline $30 \%$ & 2.03 & 0.67 & 0.44 & 3.54 & 0.38 & 0.83 \\
\hline Cruise- 43\% & 4.35 & 1.37 & 0.95 & 7.58 & 0.78 & 1.71 \\
\hline $\begin{array}{l}\text { Max Cont.- } \\
74 \%\end{array}$ & 4.68 & 1.46 & 1.02 & 8.16 & 0.83 & 1.82 \\
\hline Top-100\% & 4.68 & 1.46 & 1.02 & 8.16 & 0.83 & 1.82 \\
\hline
\end{tabular}

Table 18. Parameter of APS $3200 \mathrm{~V}=0.44 \mathrm{Ma}, \mathrm{H}=2000 \mathrm{~m}$

\begin{tabular}{|l|c|c|c|c|c|c|}
\hline & $\begin{array}{c}\text { Pressure } \\
\text { ratio }\end{array}$ & $\begin{array}{c}\text { Reduced } \\
\text { mass } \\
\text { flow }\end{array}$ & $\begin{array}{c}\text { Absolute } \\
\text { pressure } \\
\text { ratio }\end{array}$ & $\begin{array}{c}\text { APS 3200 } \\
\text { fan pressure } \\
\text { ratio }\end{array}$ & $\begin{array}{c}\text { Absolute } \\
\text { mass flow }\end{array}$ & $\begin{array}{c}\text { APS 3200 } \\
\text { fan mass } \\
\text { flow }\end{array}$ \\
\hline Idle-0\% & 0.42 & 0.12 & 0.09 & 0.72 & 0.07 & 0.16 \\
\hline $30 \%$ & 1.00 & 0.40 & 0.22 & 1.75 & 0.23 & 0.50 \\
\hline Cruise- 43\% & 4.07 & 1.41 & 0.89 & 7.09 & 0.80 & 1.76 \\
\hline $\begin{array}{l}\text { Max Cont.- } \\
74 \%\end{array}$ & 4.50 & 1.54 & 0.98 & 7.83 & 0.87 & 1.92 \\
\hline Top-100\% & 4.49 & 1.53 & 0.98 & 7.83 & 0.87 & 1.92 \\
\hline
\end{tabular}

Table 19. Parameter of APS $3200 \mathrm{~V}=0 \mathrm{Ma}, \mathrm{H}=7000 \mathrm{~m}$

\begin{tabular}{|l|c|c|c|c|c|c|}
\hline & $\begin{array}{c}\text { Pressure } \\
\text { ratio }\end{array}$ & $\begin{array}{c}\text { Reduced } \\
\text { mass } \\
\text { flow }\end{array}$ & $\begin{array}{c}\text { Absolute } \\
\text { pressure } \\
\text { ratio }\end{array}$ & $\begin{array}{c}\text { APS 3200 } \\
\text { fan pressure } \\
\text { ratio }\end{array}$ & $\begin{array}{c}\text { Absolute } \\
\text { mass flow }\end{array}$ & $\begin{array}{c}\text { APS 3200 } \\
\text { fan mass } \\
\text { flow }\end{array}$ \\
\hline Idle-0\% & 2.28 & 0.39 & 0.50 & 3.97 & 0.22 & 0.49 \\
\hline $30 \%$ & 2.29 & 0.39 & 0.50 & 4.00 & 0.22 & 0.49 \\
\hline Cruise- 43\% & 4.54 & 0.75 & 0.99 & 7.91 & 0.42 & 0.93 \\
\hline $\begin{array}{l}\text { Max Cont.- } \\
74 \%\end{array}$ & 5.05 & 0.83 & 1.10 & 8.80 & 0.47 & 1.03 \\
\hline Top-100\% & 5.05 & 0.82 & 1.10 & 8.80 & 0.47 & 1.03 \\
\hline
\end{tabular}

Table 20. Parameter of APS $3200 \mathrm{~V}=0.2 \mathrm{Ma}, \mathrm{H}=7000 \mathrm{~m}$

\begin{tabular}{|l|c|c|c|c|c|c|}
\hline & $\begin{array}{c}\text { Pressure } \\
\text { ratio }\end{array}$ & $\begin{array}{c}\text { Reduced } \\
\text { mass } \\
\text { flow }\end{array}$ & $\begin{array}{c}\text { Absolute } \\
\text { pressure } \\
\text { ratio }\end{array}$ & $\begin{array}{c}\text { APS 3200 } \\
\text { fan pressure } \\
\text { ratio }\end{array}$ & $\begin{array}{c}\text { Absolute } \\
\text { mass flow }\end{array}$ & $\begin{array}{c}\text { APS 3200 } \\
\text { fan mass } \\
\text { flow }\end{array}$ \\
\hline Idle-0\% & 2.24 & 0.39 & 0.49 & 3.91 & 0.22 & 0.49 \\
\hline $30 \%$ & 2.21 & 0.39 & 0.48 & 3.85 & 0.22 & 0.48 \\
\hline Cruise- 43\% & 4.60 & 0.78 & 1.00 & 8.01 & 0.44 & 0.97 \\
\hline $\begin{array}{l}\text { Max Cont.- } \\
74 \%\end{array}$ & 4.94 & 0.83 & 1.08 & 8.62 & 0.47 & 1.03 \\
\hline Top-100\% & 4.94 & 0.83 & 1.08 & 8.61 & 0.47 & 1.03 \\
\hline
\end{tabular}


Table 21. Parameter of APS $3200 \mathrm{~V}=0.44 \mathrm{Ma}, \mathrm{H}=7000 \mathrm{~m}$

\begin{tabular}{|c|c|c|c|c|c|c|}
\hline & $\begin{array}{c}\text { Pressure } \\
\text { ratio }\end{array}$ & $\begin{array}{c}\text { Reduced } \\
\text { mass } \\
\text { flow }\end{array}$ & $\begin{array}{c}\text { Absolute } \\
\text { pressure } \\
\text { ratio }\end{array}$ & $\begin{array}{c}\text { APS 3200 } \\
\text { fan pressure } \\
\text { ratio }\end{array}$ & $\begin{array}{c}\text { Absolute } \\
\text { mass flow }\end{array}$ & $\begin{array}{c}\text { APS 3200 } \\
\text { fan mass } \\
\text { flow }\end{array}$ \\
\hline Idle-0\% & 1.10 & 0.23 & 0.24 & 1.91 & 0.13 & 0.29 \\
\hline $30 \%$ & 1.06 & 0.23 & 0.23 & 1.85 & 0.13 & 0.28 \\
\hline Cruise- 43\% & 4.44 & 0.82 & 0.97 & 7.73 & 0.47 & 1.03 \\
\hline $\begin{array}{c}\text { Max Cont.- } \\
74 \%\end{array}$ & 4.80 & 0.88 & 1.05 & 8.37 & 0.50 & 1.10 \\
\hline Top-100\% & 4.80 & 0.88 & 1.05 & 8.37 & 0.50 & 1.10 \\
\hline
\end{tabular}

All obtained parameters are plotted in Fig.6.

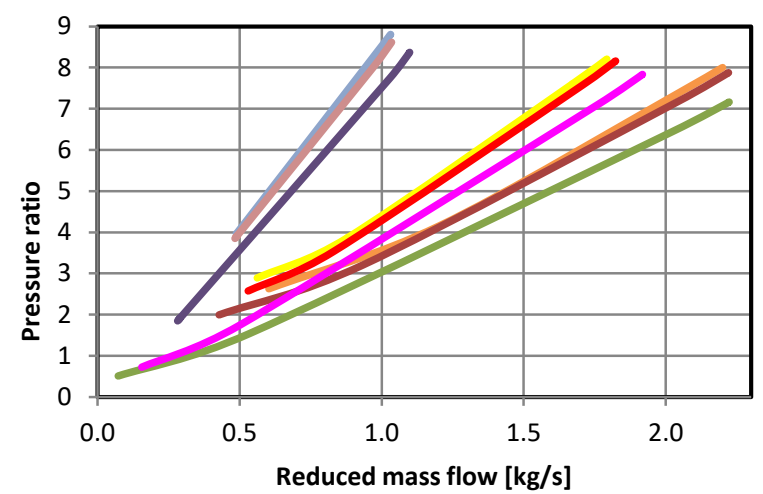

$$
\begin{aligned}
& \longrightarrow \mathrm{V}=0 \mathrm{Ma} ; \mathrm{H}=\mathrm{Om} \longrightarrow \mathrm{V}=\mathrm{OMa} ; \mathrm{H}=2000 \mathrm{~m} \quad \mathrm{~V}=\mathrm{OMa} ; \mathrm{H}=7000 \mathrm{~m} \\
& -\mathrm{V}=0,2 \mathrm{Ma} ; \mathrm{H}=\mathrm{Om} \longrightarrow \mathrm{V}=0,2 \mathrm{Ma} ; \mathrm{H}=2000 \mathrm{~m} \longrightarrow \mathrm{V}=0,2 \mathrm{Ma} ; \mathrm{H}=7000 \mathrm{~m} \\
& -\mathrm{V}=0,44 \mathrm{Ma} ; \mathrm{H}=\mathrm{Om} \_\mathrm{V}=0,4 \mathrm{Ma} ; \mathrm{H}=2000 \mathrm{~m} \quad-\mathrm{V}=0,44 \mathrm{Ma} ; \mathrm{H}=7000 \mathrm{~m}
\end{aligned}
$$

Fig. 6. Compressor map of centrifugal compressor APS 3200

The compressor map of centrifugal compressor of APS 3200 has got characteristic division into three groups like the lines of fan of DGEN 380 and CFM 56-5B. Exactly as in the previous cases it is caused by unstable parameters of flow. Despite the fact this compressor map is plotted based on parameters of compressor in DGEN 380, which is preceded by fan stabilizing the flow, the chart of centrifugal compressor APS 3200 exhibits features of the division into three group, exactly like maps of DGEN 380 fan and CFM 56-5B fan. In fact compressor of APS 3200 is not preceded by any components, which could stabilize the parameters. It means that engine analogy applicable in practice.

\section{Conclusion}

Obtained data getting by using virtual bench test WESTT CS/BV were tabulated. The mass flow was reduced using equation (1) for data of fan and compressor. Because of that the charts are not dependent on ambient conditions, that were necessary because they have huge impact on parameters of compressor.

Getting data by using virtual bench test allowed plotting characteristic of fan and compressor very accurately. On the compressor map is visible:

- the increase of flight velocity causes: the increase of component mass flow and also when the rotational speed is low it causes low efficiency of both components,

- the increase of altitude flight causes move the lines in stall margin direction,

- stabilized parameters at inlet of components causes greater similarity of lines on the different altitude of flight.

Using MATLAB code and mathematical equations, which are equations of function above lines there is opportunity to plotted compressor map of engines with components with the same construction of compressor or fan. To mapping mentioned characteristic using the engine analogy the reduction of parameters (pressure ratio and mass flow) to Absolute is necessary.

The engine analogy results are correct, what confirms the compressor map of centrifugal compressor of APS 3200. The lines on it are ordered similar to the compressor map of fan DGEN 380. Despite using the equations obtained from the compressor map of DGEN 380, which parameters of flow are stable, the chart of compressor map of centrifugal compressor of APS 3200 do not show characteristics of uniform flow parameters. It is adequate to its construction, because the compressor of APS 3200 is not preceded by any component, which could uniform the parameters.

Obtained functions of lines for the fan and the compressor may be used to preliminary engine design.

\section{Nomenclature}

\section{$\dot{m}$ mass flow}

$\dot{\mathrm{m}}_{\text {max }}$ maximal mass flow at sea level

$\dot{\mathrm{m}}_{\text {abs }}$ absolute mass flow

$\dot{\mathrm{m}}_{\mathrm{r}}$ reduced mass flow

$\pi \quad$ pressure ratio $\pi_{\max }$ maximal pressure ratio an sea-level

$\pi_{\mathrm{abs}}$ absolute pressure ratio

$\mathrm{p}_{0} \quad$ ambient pressure

$\mathrm{T}_{0} \quad$ ambient temperature

\section{Bibliography}

[1] SAVARANAMUTTO, H.I.H., ROGERS, G.F.C., COHEN, H. Gas Turbine Theory. 5 edition, Pearson, 2013, 178, 256.

[2] DRUMMOND, C., DAVISON, C.R. Improved compressor maps using approximate solutions to the Moore-Greitzer model. Volume 1: Aircraft Engine; Ceramics; Coal, Biomass and Alternative Fuels; Controls, Diagnostics and Instrumentation; Education; Electric Power; Awards and Honors. https://doi.org/10.1115/gt2009-60148
[3] HUNZKIER, R., DICKMANN, H-P., EMMRICH, R. Numerical and experimental investigation of a centrifugal compressor with an inducer casing bleed system. Proceedings of the Institution of Mechanical Engineers, Part A: Journal of Power and Energy. 2001, 215(6), 783-791. https://doi.org/10.1243/0957650011538910

[4] KURZKE, J., RIEGLER, C. A new compressor map scaling procedure for preliminary conceptional design of gas tur- 
bines. Volume 1: Aircraft Engine; Marine; Turbomachinery; Microturbines and Small Turbomachinery.

https://doi.org/10.1115/2000-gt-0006

[5] KURZKE, J. Correlations hidden in compressor maps. Volume 1: Aircraft Engine; Ceramics; Coal, Biomass and Alternative Fuels; Wind Turbine Technology. https://doi.org/10.1115/gt2011-45519

[6] ISMAIL, I.H., BHINDER, F.S. Simulation of aircraft gasturbine engine. ASME, Journal of Engineering for Gas Turbines and Power. 1991, 95-99.

https://doi.org/10.1115/1.2906536

[7] ELGAMMAL, M.A. An algorithm and criteria for compressor characteristics real time modelling and approximation. ASME. Journal of Engineering for Gas Turbines and Power. 1991, 112-117. https://doi.org/10.1115/1.2906517

[8] SIEROS, G., STAMATIS, A., MATHIOUDAKIS, K. Jet engine component maps for performance modelling and diagnosis. AIAA, Journal of Propulsion and Power. 1997, 13(5), 665-674. https://doi.org/10.2514/2.5218

Karolina Pazura, MEng. - graduate Faculty of Mechanical Engineering and Aeronautics, Rzeszow University of Technology.

e-mail: k.pazura.kp@gmail.com
[9] MORAAL, P., KOLMANOSKY, I. Turbocharger modelling for automotive control application. SAE Technical Paper 1999-01-0908. 1999. https://doi.org/10.4271/1999-01-0908

[10] KONG, C.D., KHO, S., KI, J.Y. Component map generation of a gas turbine using genetic algorithms. ASME. Journal of Engineering for Gas Turbines and Power. 2006, 128(1), 9296. https://doi.org/10.1115/1.2032431

[11] KONG, C.D., KI, J.Y. Components map generation of gas turbine engine using genetic algorithms and engine performance deck data. ASME. Journal of Engineering for Gas Turbines and Power. 2007, 129(2), 312-317.

https://doi.org/10.1115/1.2436561

[12] GRAVDAHL, J.T., EGELAND, O. Centrifugal compressor surge and speed control. IEEE Transactions on Control Systems Technology. 1999, 7(5), 567-579. https://doi.org/10.1109/87.784420

[13] GRAVDAHL, J.T., EGELAND, O., WILLEMS, F., JAGER B.D.E. Modeling of surge in free-spool centrifugal compressors: Experimental validation. AIAA. Journal of Propulsion and Power. 2004, 20(5), 849-857. https://doi.org/10.2514/1.10052

Prof. Marek Orkisz, DSc., DEng. - Faculty of Mechanical Engineering and Aeronautics Rzeszow University of Technology.

e-mail:mareko@prz.edu.pl 